\title{
Utilização de SPI e CPI no acompanhamento estatístico de projetos de desenvolvimento de software
}

\author{
Renata L. Freitas ${ }^{1}$, Sandra C. Moreira ${ }^{1}$, Cintya C. Corgosinho Suzuki ${ }^{1}$, Lucilia V. \\ Moreira $^{1}$, Priscilla N. Romeu ${ }^{1}$, Rosana V. Xavier ${ }^{2}$, Pedro Lopes da Rocha Leal Jr. ${ }^{1}$ \\ ${ }^{1}$ Spread Systems - Unidade MSA-Infor \\ R. Andaluzita, 110 - 30.310-030 - Belo Horizonte - MG - Brasil \\ ${ }^{2}$ PPGEE - Programa de Pós-Graduação em Engenharia Elétrica - PUCMG - Pontifícia \\ Universidade Católica de Minas Gerais - Av. Dom José Gaspar, 500 - 30535-901 - \\ Belo Horizonte - MG - Brasil \\ \{renata.freitas, sandra.moreira, cintya, lucilia.moreira, \\ priscilla.neves, pedro\}@spread.com.br, rosanavx@gmail.com
}

Resumo. Este relato descreve a experiência da Spread Systems - Unidade MSA-Infor em utilizar os indicadores SPI e CPI, definidos pela técnica de Análise de Valor Agregado, no acompanhamento de projetos de desenvolvimento de software. São apresentadas as necessidades de adaptação do SPI e CPI, a utilização de ferramentas estatísticas de controle de processo no acompanhamento destes indicadores, os resultados obtidos, principais dificuldades e lições aprendidas.

\begin{abstract}
This paper describes the experience of the Spread Systems - Unit MSA-Infor using the SPI and CPI indicators, defined by the technique of Earned Value Analysis, to monitor and control software development projects. It presents the adaptation needs of the SPI and CPI, the use of statistical tools for process control in monitoring these indicators, the results obtained, the main difficulties and lessons learned.
\end{abstract}

\section{Introdução}

Para alcançar os níveis mais altos de maturidade do modelo $\mathrm{CMMI}^{\circledR}$-DEV as organizações devem estabelecer objetivos quantitativos para a qualidade dos produtos e para o desempenho dos processos nos projetos de desenvolvimento de software. A qualidade e o desempenho dos processos devem ser controlados por meio de técnicas estatísticas e gerenciados ao longo do ciclo de vida do processo [Paulk e Chrissis 2001], com o objetivo de conseguir previsibilidade dos resultados [Chrissis 2007].

Como forma de acompanhar estatisticamente seus processos, as organizações fornecedoras de software vêem adotando técnicas de controle estatístico de processo CEP [Montgomery 2004], amplamente utilizadas na indústria [Sargut 2006], porém as características inerentes de um produto de software, como complexidade, conformidade, instabilidade e invisibilidade [Brooks 1987], dificultam a aplicação destas técnicas em processos de desenvolvimento de software.

Dentro deste contexto, este relato tem como objetivo descrever a experiência da Spread Systems - Unidade MSA Infor em utilizar os indicadores SPI e CPI, definidos 
pela técnica de análise do valor agregado, no acompanhamento dos projetos de desenvolvimento de software. Serão apresentadas a derivação dos objetivos estratégicos da organização em metas setoriais e a definição de indicadores de desempenho que, analisados através de ferramentas estatísticas, permitem verificar se a execução dos projetos está coerente com o planejado, em termos de prazo e esforço. Este relato está estruturado em 3 seções, além desta introdução. Na seção 2 são apresentados a organização e os conceitos e técnicas utilizados. Na seção 3 é detalhada a experiência em utilizar técnicas estatísticas para o acompanhamento de indicadores de desempenho e, na seção 4, são apresentadas as lições aprendidas e considerações finnais.

\section{Contextualização}

\subsection{A empresa}

Fundada em 1983, a Spread atua no mercado brasileiro de tecnologia da informação, oferecendo soluções integradas nos segmentos de IT Outsourcing \& BPO, Telecom \& Network e Systems. Para complementar a sua atuação no segmento de sistemas a Spread adquiriu, em 2008, a MSA Infor, empresa com mais de 20 anos de experiência no desenvolvimento e integração de produtos e serviços de tecnologia da informação.

A Spread Systems - Unidade MSA Infor possui uma constante preocupação com a qualidade e, desde sua fundação, vem evoluindo seus processos através da adoção de conceitos e práticas bem sucedidas de mercado como o RUP ${ }^{\circledR}, I_{S O}$, PMBOK $^{\circledR}$ e $\mathrm{CMMI}^{\circledR}$. Em 2010 a Spread Systems - Unidade MSA Infor alcançou o nível 5 do modelo CMMI ${ }^{\circledR}$-DEV, V1.2, mais alto nível de maturidade do modelo.

\subsection{CMMI®}

O CMMI ${ }^{\circledR}$ (Capability Maturity Model ${ }^{\circledR}$ Integration) é um modelo de maturidade para melhoria de processos composto pelas melhores práticas de desenvolvimento e manutenção de produtos e serviços. A estrutura atual do CMMI® suporta duas representações: representação por estágios e representação contínua. A representação contínua oferece uma abordagem de melhoria incremental dos processos de uma determinada área. Já a representação por estágios possibilita a melhoria de um conjunto de áreas de processos associadas a um determinado nível de maturidade.

Nos níveis mais altos de maturidade as organizações são capazes de analisar as causas de variações inerentes aos processos de forma a estabilizá-los e torná-los mais previsíveis quanto aos resultados esperados. Com a estabilização dos processos é possível implantar melhorias incrementais e inovadoras de processo e tecnologia de forma a alcançar os objetivos estabelecidos [Chrissis 2007].

\subsection{Ferramentas estatísticas para controle de processos}

O Controle Estatístico de Processo (CEP) é uma área do controle da qualidade de produtos e serviços que consiste na coleta e análise de dados com o objetivo de identificar defeitos nos processos avaliados. As cartas de controle de Shewhart são as principais ferramentas utilizadas no CEP para o monitoramento da estabilidade e da variação de um processo [Werkema 1995]. As cartas de controle permitem a observação da existência de causas de variação no processo. Estas variações podem representar uma situação particular que faz com que o processo se comporte de maneira diferente do usual. De modo geral, estas causas devem ser localizadas e eliminadas e medidas corretivas devem ser tomadas para evitar sua reincidência. 


\subsection{Técnica de Análise do valor agregado}

A técnica de análise do valor agregado consiste na comparação valor agregado (VA) com o valor planejado (VP) e com custo real (CR) em um determinado momento [PMI 2004]. Estes valores, utilizados em conjunto, permitem estabelecer índices de desempenho que indicam se o trabalho está sendo realizado conforme o planejado. $\mathrm{O}$ valor agregado pode ser analisado através de três curvas de desempenho que representam estes valores, como mostrado na figura 1. A situação representada neste exemplo exibe um projeto que se encontra acima do orçamento e atrasado em relação ao plano de trabalho.

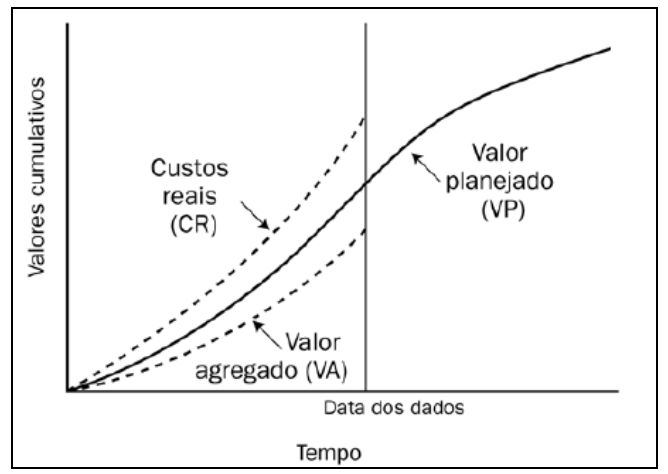

Figura 1 - Curvas de desempenho

Adaptado de: PMI, 2004, p.174.

Os principais indicadores de desempenho são o Schedule Performance Index (SPI) e Cost Performance Index (CPI). O SPI representa a medida de eficiência do cronograma, dado pela razão entre o VA e o VP [PMI 2004]. Um projeto que cumpre exatamente os prazos planejados apresenta SPI igual a um. O CPI é uma medida da eficiência de custos, em um projeto, dado pela razão entre o VA e o CR [PMI 2004]. Um CPI igual a um indica que o projeto está com os custos iguais aos previstos no orçamento.

\section{Descrição da Experiência}

Os objetivos organizacionais da Spread Systems - Unidade MSA Infor são alinhados ao planejamento estratégico da organização e são representados por três grandes pilares: Crescer, Rentabilizar e Perpetuar. Cada um destes pilares foi derivado em metas relacionadas às necessidades de cada uma das áreas da unidade.

As metas foram apresentadas ao Software Engineering Process Group (SEPG), grupo responsável por avaliar, apresentar e implantar novos métodos e tecnologias que visam à melhoria dos processos de desenvolvimento de software da organização e tratado como Engineering Process Group (EPG) no modelo CMMI ${ }^{\circledR}$ [Chrissis 2007]. Segundo Lantzy, o controle estatístico de processos deve ser aplicado apenas em processos críticos, ou seja, os sub-processos que afetavam diretamente, ou com maior intensidade, os objetivos e metas organizacionais [Lantzy 1992]. O SEPG realizou o mapeamento dos processos críticos e estabeleceu indicadores para o acompanhamento de cada processo mapeado. Tais indicadores permitiam verificar que os objetivos definidos estavam sendo alcançados nos projetos e, conseqüentemente, por toda a organização. Este mapeamento foi realizado utilizando a metodologia Goal Question 
Metrics [Basili 1994]. O quadro 1 apresenta a derivação dos objetivos organizacionais nos objetivos de medição apresentados neste artigo.

\section{Quadro 1}

Derivação dos objetivos organizacionais em objetivos de medição

\begin{tabular}{|c|c|c|c|c|c|}
\hline $\begin{array}{c}\text { Objetivo } \\
\text { organizacional }\end{array}$ & Meta & Sub-meta & $\begin{array}{c}\text { Questão (Requisito de } \\
\text { informação) }\end{array}$ & Processo crítico & Indicador \\
\hline $\begin{array}{l}\text { Crescer e } \\
\text { Perpetuar }\end{array}$ & $\begin{array}{c}\text { Fomentar } \\
\text { demandas } \\
\text { Competência } \\
\text { Organizacional }\end{array}$ & $\begin{array}{l}\text { Entregar } \\
\text { no prazo }\end{array}$ & $\begin{array}{l}\text { Os pontos de controle } \\
\text { (incluindo o prazo } \\
\text { final) estão } \\
\text { acontecendo na data } \\
\text { prevista? }\end{array}$ & $\begin{array}{c}\text { Acompanhar a } \\
\text { execução do } \\
\text { planejamento }\end{array}$ & SPI \\
\hline
\end{tabular}

\subsection{Adaptação do CPI para EPI}

Um dos principais processos críticos identificado está relacionado ao acompanhamento da execução do planejamento, visando reduzir o desvio de esforço entre o planejado e o realizado. Para acompanhar o desempenho deste processo foram estabelecidos os indicadores de prazo e esforço. O EPG considerou que os custos do projeto deveriam ser analisados em termos de homem-hora, o que gerou a necessidade de adaptação do indicador de custo. Com isso as horas de trabalho passaram a ser utilizadas como unidade de medida para o VA e o CR, o que tornou o CPI um índice de desvio de esforço, o Effort Performance Index (EPI). O EPI representa então o esforço total (em horas) efetivo contra o previsto para o avanço atual do projeto. Um projeto que cumpre exatamente os esforços planejados apresenta um EPI igual a 1.

\subsection{O acompanhamento do SPI e EPI utilizando ferramentas estatísticas para o controle de processos}

O gerente do projeto é responsável por elaborar um planejamento que englobe as atividades de desenvolvimento do projeto e os responsáveis por essas atividades, para posteriormente serem alocados os recursos humanos necessários para cada uma delas. $\mathrm{O}$ cronograma do projeto é elaborado conforme as atividades, recursos e esforços planejados. Para elaboração e acompanhamento do cronograma utilizou-se a ferramenta MS Project 2003. Após aprovação do cronograma é gerada uma linha de base do planejamento [Vargas 2004]. A linha de base é o parâmetro inicial que o gerente do projeto utiliza para acompanhar o planejamento.

Através do MS Project Server [Vargas 2004] os recursos possuem visibilidade de todas as atividades a eles atribuídas. À medida que as atividades são executadas os recursos informam as horas gastas na sua execução, através da interface web da ferramenta. $\mathrm{O}$ gerente do projeto aprova as horas informadas e estas passam a ser contabilizadas como custo real e utilizadas no cálculo do valor agregado.

Todas as métricas e indicadores estatísticos da organização são analisados utilizando uma planilha eletrônica chamada AMP (Acompanhamento das Métricas do Projeto). Para viabilizar a utilização desta planilha na análise dos indicadores SPI e EPI, realizou-se a automação da coleta dos valores de VP, VA e CR, diretamente da base de dados do MS Project, para atualização da planilha AMP com os valores coletados. Nesta 
planilha foram implantadas as cartas de controle e a identificação automática dos pontos que configuram uma causa especial de variação, viabilizando o acompanhamento estatístico dos indicadores.

A coleta dos valores de VP, VA e CR e a análise do SPI e do EPI são realizadas semanalmente pelo gerente de projeto. Cada semana representa um ponto na carta de controle. A análise consiste em justificar a variação do indicador, quando significativa, e compará-lo ao comportamento e metas da organização. A comparação com o comportamento organizacional permite identificar a existência de causas especiais que são investigadas na tentativa de encontrar sua causa raiz e tomar ações para evitar sua reincidência. $\mathrm{O}$ mesmo procedimento pode ser aplicado quando o índice da semana está fora da meta da organização.

A ferramenta utilizada para o acompanhamento do cronograma fornece os valores de VP, VA e CR que são utilizados no cálculo do SPI e EPI e analisados semanalmente pelo gerente do projeto. Porém, os valores fornecidos pela ferramenta são valores acumulados que, quando utilizados em uma carta de controle, podem indicar tendências de comportamento que configurariam "alarmes falsos" de causas especiais. No gráfico 1 observamos a existência da causa especial D que configura nove pontos consecutivos de um mesmo lado do valor da média de controle estatístico.

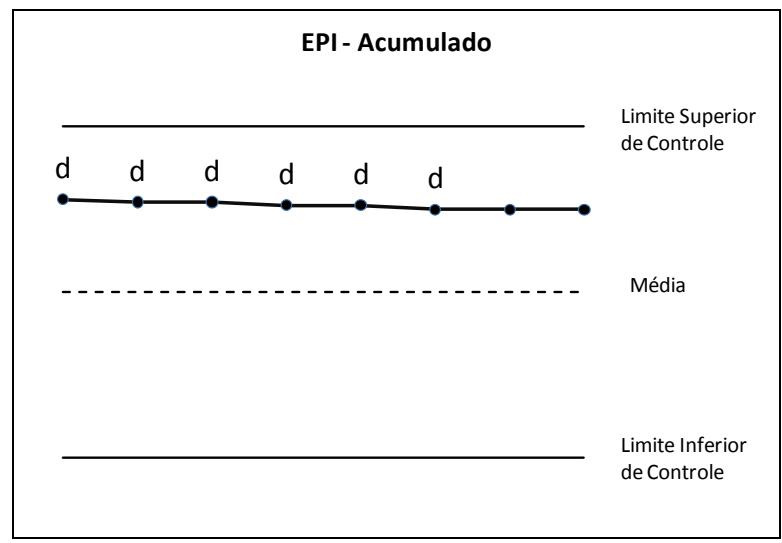

Gráfico 1 - Carta de controle com presença de causas especiais de variação.

Com o decorrer do projeto os valores se acumulam e as atividades realizadas na semana se tornam pouco significativas em relação ao todo, levando a pequenas variações dos indicadores e, conseqüentemente, ao surgimento de tendências. Tal comportamento viola a suposição de independência dos valores a serem inseridos nas cartas convencionais de Shewhart [Mingoti e Yassukawa 2008]. Portanto, essas cartas não poderiam ser utilizadas para realização do acompanhamento estatístico destes indicadores, sendo necessária a utilização de outras ferramentas estatísticas.

As ferramentas aplicáveis a este caso poderiam dificultar a análise e a sua implantação poderia ser mais complexa, visto que os envolvidos já estavam bem familiarizados com as cartas convencionais de Shewhart. Como solução para o problema foi realizada uma adaptação nos valores fornecidos pelo cronograma para que as cartas de controle pudessem ser utilizadas de forma correta. As atividades passaram a ser acompanhadas em um maior nível de detalhe considerando a variação ocorrida dentro de uma semana no projeto, complementando a análise estatística feita através dos 
indicadores acumulados. Com isso, os índices de SPI e EPI acumulados deixaram de ser monitorados em relação a sua estabilidade e passaram a ser acompanhados em um gráfico seqüencial, dando assim ao gerente uma visão do projeto como um todo.

Para a obtenção dos valores de VP, VA e CR semanais, foi realizada uma operação matemática simples: subtrair os dados acumulados da semana em análise dos dados acumulados da semana anterior, para se obter a variação na semana de interesse. Esta adaptação também foi automatizada na planilha AMP para facilitar as análises pelos gerentes de projeto.

Para o acompanhamento dos projetos foi utilizada a carta de controle para dados individuais X-AM [Werkema 1995]. A utilização desta carta exige que a suposição de normalidade para os dados seja satisfeita. Uma variável tem distribuição normal se essa distribuição é simétrica e apresenta uma forma de sino [Triola 1999]. Ao inserir o valor correspondente à variação semanal na carta de controle observou-se que os mesmos não eram simétricos. Considerando um valor agregado igual a 25 e um valor planejado igual a 1, obtêm-se um SPI igual a 25 indicando que o projeto está adiantado. Ao considerar o contrário, um valor agregado igual a 1 e um valor planejado igual a 25 , obtêm-se um SPI igual a 0,04 indicando que o projeto está atrasado na mesma proporção. Porém, ao inserir esses dois valores em uma mesma carta de controle observa-se que eles não são simétricos e que apenas o ponto igual a 25 configura uma causa especial, como mostrado no gráfico 2 . O ponto igual a 0,04 também deveria ser configurado como uma causa especial na mesma proporção do ponto igual a 25 , o que não ocorreu devido à falta de simetria do indicador.

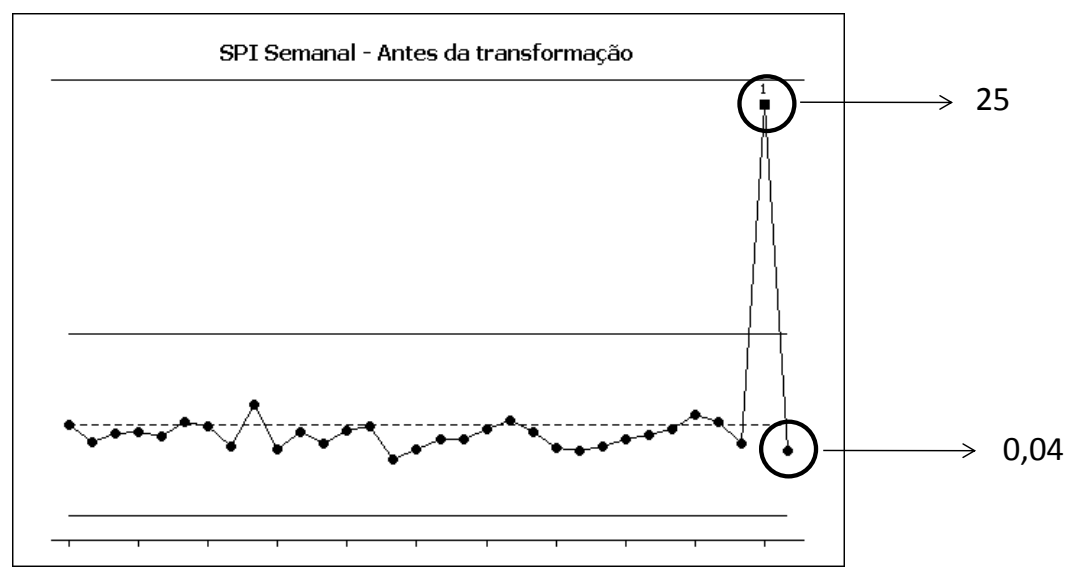

\section{Gráfico 2 - SPI Semanal - Antes da transformação: os dois pontos deveriam configurar uma causa especial.}

Para garantir a simetria dos dados, os mesmos foram submetidos a uma transformação usando a função logarítmica na base 2. Vale lembrar que a função logarítmica trata os números e seus recíprocos simetricamente [Quackenbush 2002], por exemplo: $\log _{2}(2)=1, \log _{2}(1 / 2)=-1, \log _{2}(8)=3, \log _{2}(1 / 8)=-3$. O valor de comparação também deve acompanhar a escala dos dados transformados, portanto os valores do SPI e EPI semanais não seriam mais comparados ao valor 1 , e sim ao valor 0 , pois $\log _{2}(1)=$ 0 . Com a aplicação da função logarítmica, os dois pontos exemplificados são simétricos e ambos configuram causas especiais, como mostrado no gráfico 3. 


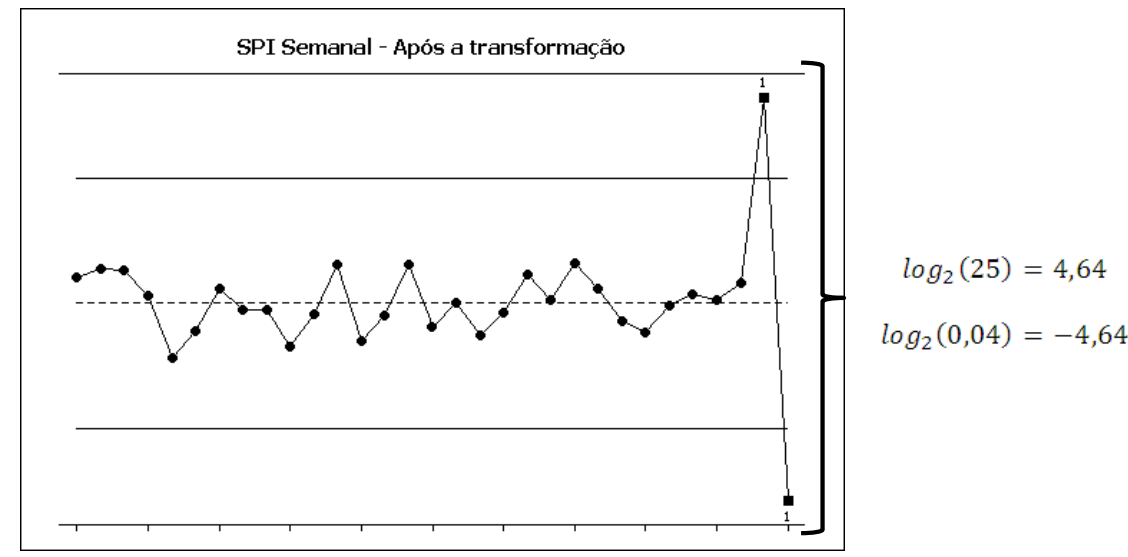

Gráfico 3 - SPI Semanal - Após a transformação: os dois pontos configuram uma causa especial.

\section{Lições aprendidas e considerações finais}

O principal desafio enfrentado na implantação dos indicadores SPI e EPI na organização foi a necessidade de mudança cultural na gestão dos projetos. $\mathrm{O}$ acompanhamento semanal dos indicadores exigiu uma dedicação maior por parte dos gerentes de projeto que, inicialmente, demonstraram resistência aos novos procedimentos. $\mathrm{O}$ grande motivador para a institucionalização foi a busca pelo mais alto nível de maturidade do modelo $\mathrm{CMMI}^{\circledR}$-DEV que estabelece práticas de acompanhamento estatístico como forma de verificar se os objetivos organizacionais estão sendo alcançados. Como os gerentes não tinham um conhecimento aprofundado das ferramentas estatísticas, foi de extrema importância a realização de treinamentos específicos e a designação de um profissional especializado para apoiá-los nas análises.

Com a adequada utilização da carta de controle, o gerente do projeto pôde avaliar as atividades planejadas, o valor agregado e o custo real apenas dentro da semana em análise e, quando necessário, identificar as causas dos desvios ocorridos e tomar ações corretivas e preventivas. O acompanhamento mais detalhado permitiu à organização conhecer melhor o comportamento de seus projetos e eliminar possíveis desvios que afetariam negativamente seus prazos e esforços finais.

Pôde-se observar também que o acompanhamento semanal facilitou a identificação de necessidades de replanejamentos de um projeto de forma a reverter situações de atrasos e/ou desvios de esforço. Após algumas semanas de acompanhamento identificou-se que os indicadores semanais não deveriam ser coletados em semanas de replanejamentos, pois os mesmos podem sofrer grandes variações devido ao remanejamento das atividades. Desta forma, recomenda-se que nestas semanas, apenas os valores acumulados de SPI e EPI sejam analisados.

Vale ressaltar que a análise dos indicadores semanais não substituiu a análise dos indicadores acumulados, uma vez que os indicadores semanais fornecem um retrato mais detalhado do projeto, enquanto os índices acumulados dão uma visão geral do projeto como um todo e da tendência de seu comportamento.

A experiência da Spread Systems mostrou que a carta de controle pode ser um importante aliado no acompanhamento semanal dos projetos. Apesar de ainda não terem sido coletados dados suficientes para avaliar quantitativamente os resultados do 
acompanhamento estatístico dos indicadores, foi possível perceber de forma qualitativa os benefícios que esta prática proporcionou para a gestão dos projetos na organização.

\section{Referências}

Basili, V.; Caldiera, G. \& Rombach, H. (1994). "The goal question metric approach". Encyclopedia of software engineering, 1:528-532.

Brooks, F.P. (1987). "No Silver Bullet: Essence and Accidents of Software Engineering", IEEE Computer Magazine.

Chrissis, M.B., Konrad, M. e Shrum, S. (2007) "CMMI: guidelines for process integration and product improvement". Addison-Wesley

Lantzy, M.A., (1992) "Application of Statistical Process Control to Software Processes", WADAS '92. Proceedings of the Ninth Washington Ada Symposium on Empowering Software Users and Developers. P 113-123.

Mingoti, S. A., Yassukawa F. R. S. (2008) "Uma comparação de gráficos de controle para a média de processos autocorrelacionados" Revista Eletrônica Sistemas \& Gestão 3 (1) 55-73. Programa de Pós-graduação em Sistemas de Gestão, TEP/TCE/CTC/PROPP/UFF.

Montgomery, D. C. (2004). “Introdução ao Controle Estatístico da Qualidade”. Livros Técnicos e Científicos Editora S.A.

Paulk, M.C., Chrissis, M.B. (2001) "The 2001 High Maturity. Workshop" (CMU/SEI2001-SR-014). Pittsburgh, PA: Software. Engineering Institute, Carnegie Mellon University, January 2002.

PMI (2004). "Um Guia do Conjunto de Conhecimentos em Gerenciamento de Projetos (Guia PMBOK)" Terceira edição. Project Management Institute, Four Campus Boulevard, Newtown Square, PA 19073-3299 EUA.

Quackenbush, J. (2002) "Microarray data normalization and transformation". Nat Genet, 32:496 -501.

Sargut, K. U., Demirors, O. (2006) "Utilization of Statistical Process Control (SPC) in Emergent Software Organizations: Pitfalls and Suggestions", Software Quality Journal, v. 14, n. 5, p. 135-157.

Triola (1999), M. F. "Introdução a estatística". 7. Edição, Rio de Janeiro. LTC, p113

Vargas, R. V. (2004). “Microsoft Office Project 2003”. Standard, Professional \& Server. Brasport

Werkema, M. C. C. (1995) "Ferramentas estatísticas básicas para o gerenciamento de processos". Serie Ferramentas da Qualidade. Belo Horizonte, UFMG, EE/F.C.O., $384 p$. 